\title{
Characterization of Sensor Performance and Durability for Structural Health Monitoring Systems
}

\author{
James L. Blackshire ${ }^{\mathrm{a}}$, Victor Giurgiutiu ${ }^{\mathrm{b}}$, Adam Cooney ${ }^{\mathrm{a}}$, and James Doane ${ }^{\mathrm{b}}$ \\ ${ }^{a}$ Air Force Research Lab (AFRL/MLLP), Wright-Patterson AFB, OH 45433 \\ ${ }^{\mathrm{b}}$ Department of Mechanical Engineering, University of South Carolina \\ Columbia, SC 29208, USA
}

\begin{abstract}
A key question that needs to be addressed and answered with regard to successfully implementing Structural Health Monitoring technologies in Air Force systems involves the long-term operability, durability, and survivability of integrated sensor systems and their associated hardware. Whether a sensor system is fully integrated within a structural material, or surface-bonded to the structure, a number of environmental and system level influences will tend to degrade the sensor system's performance and durability over time. In this effort, an initial sensor durability study was undertaken to better understand the performance and degradation of piezo wafer active sensor (PWAS) systems under adverse mechanical, temperature, and moisture conditions. A novel displacement-field imaging approach was utilized to understand the vibration characteristics of PWAS transducers placed in accelerated vibration, temperature-cycling, and moisture-cycling conditions. The results showed damage in the form of PWAS sensor cracking events, bond degradation and failure, as well as indications of performance variation and reduction due to the accelerated exposure levels. Future activities will focus on identifying critical durability and survivability issues through advanced sensor modeling and additional accelerated testing efforts, with the ultimate goal of improving the robustness of health monitoring systems through improved sensor system design and packaging.
\end{abstract}

Keywords: Structural Health Monitoring, Piezo Wafer Active Sensors, Displacement-Field Imaging

\section{INTRODUCTION}

Significant progress has recently been reported in the areas of structural health monitoring (SHM) and integrated systems health management (ISHM). A number of key enabling technologies are quickly coming together to provide miniaturized, autonomous, and capable sensing platforms for monitoring the structural integrity and health of a wide variety of civil, industrial, and aerospace systems ${ }^{1-4}$. Several practical considerations related to optimal sensor placement, efficient data analysis and reduction methods, and understanding the fundamental physics between sensing data and structural damage levels are also being addressed ${ }^{5-8}$. In the aerospace industry, the implications of SHM and ISHM are expected to be significant and revolutionary with respect to safe-life engineering design, condition-based maintenance, and damage prognosis assessment.

The vast majority of structural health monitoring concepts are based on integrated sensors, which are either bonded to an existing structure, or integrated directly within a newly fabricated structure ${ }^{9-11}$. These integrated sensors become a part of the structure, and are subjected to similar or identical environmental conditions. A key concern for SHM and ISHM sensors is the long term durability, survivability, and operation performance over extended time periods and in a wide variety of varying environmental conditions. The general expectation is that the sensors will behave in a consistent and reliable manner throughout the duration of the health monitoring inspection exercise. For aerospace structures, the duration of this inspection period can span several years and even decades. During such periods, the sensors should remain true to their initial condition, with changes in sensor readings being due solely to actual changes in the structural health state and condition. 
For integrated piezoelectric sensors, a number of environmental conditions can impact long-term performance and operation. In an aircraft environment, temperature, moisture, strain, load, vibrations, and corrosion/chemical attack can potentially degrade performance. Temperature cycling will, for example, lead to strain in the sensor and bond materials due to differences in coefficient of thermal expansion (CTE) between the aircraft substrate material and the piezoelectric sensor material. Bending and tensile/compressional loads will also strain the sensor material, and in extreme conditions can lead to bond failure and sensor fracture. Corrosion and other types of chemical attack (cleaning solvents, fuels, lubricants) are also likely, potentially affecting substrate bond condition, and electrical contact degradation over time. To gain widespread acceptance, SHM and ISHM sensor systems have to be proven rugged, durable, and reliable in every imaginable environmental condition expected in typical operational use.

In this effort, an initial sensor durability study was undertaken to better understand the performance and degradation of piezo wafer active sensor (PWAS) systems under adverse mechanical, temperature, and moisture conditions. A novel displacement-field imaging approach was utilized to understand the vibration characteristics of PWAS transducers placed in accelerated vibration, temperature-cycling, and moisture-cycling conditions. The results showed damage in the form of PWAS sensor cracking events, bond degradation and failure, as well as indications of performance variation and reduction due to the accelerated exposure levels. Future activities will focus on identifying critical durability and survivability issues through advanced sensor modeling and additional accelerated testing efforts, with the ultimate goal of improving the robustness of health monitoring systems through improved sensor system design and packaging.

\section{PIEZO WAFER ACTIVE SENSORS (PWAS)}

One of the most appealing sensor concepts for aerospace applications involves using integrated piezoelectric sensor concepts. Piezoelectric sensors convert mechanical energy into electrical energy and visa-versa. In an active sensing mode, the piezoelectric effect is used to general elastic wave motions in a material substrate by applying a voltage potential across the piezo sensor thickness. Damage can be detected and characterized through elastic wave reflection and scattering processes, where the amplitude, phase, and frequency content of the waves can be used to understand the extent, location, and type of damage. In a passive sensing mode, piezo sensors assess damage through acoustic emission signatures and through electromechanical (E/M) impedance measurements. More details of the active piezo sensing approach can be found in [12], while details of the acoustic emission and electromechanical impedance methods can be found in [13] and [14], respectively.

On a more practical level, the use of thin, inexpensive piezo disks, is of particular interest to SHM and ISHM sensing applications. Also referred to as piezo wafer active sensors (PWAS), these single crystal piezoelectric materials can be engineered to a wide variety of specifications for specific ISHM applications. They are typically surface-bonded to a structure as individual sensors, and in combination can be used for large area sensing or phased-array measurements. PWAS represent one of the least expensive sensor materials (\$1-\$10 per sensor), and are lightweight and minimally intrusive to the structure. Figure 1a provides a digital image and cross-sectional cut through a bonded PWAS sensor.

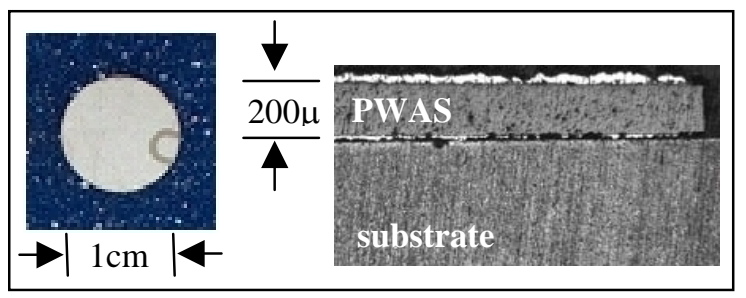

a)

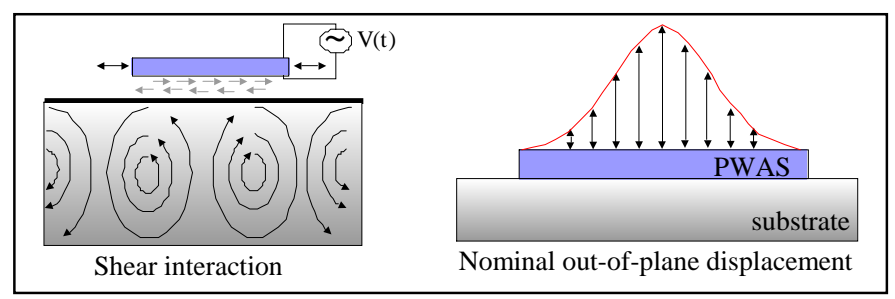

b)

Fig. 1. a) Digital image and cross-sectional cut through PWAS, b) shear layer interaction and out-of-plane displacement for PWAS.

When a voltage potential is placed across the thickness of a PWAS sensor, piezoelectric motions (expansions and contractions) are generated within the sensor material. These motion fields transfer elastic wave energy into the substrate material, which can be used to actively probe the substrate's health state. Conversely, when a motion perturbs 
the PWAS sensor and causes it to physically expand or contract, a voltage potential is generated that can be used to monitor the elastic motion fields present in the material substrate.

As depicted in Figure 1a, the PWAS chosen for this study were circular disks with diameters in the $6 \mathrm{~mm}-2 \mathrm{~cm}$ range, and a thickness of 200 microns. The piezo crystal cut of the PWAS disks was chosen to optimize Lamb wave generation and propagation in thin plate aluminum structures. In simplified terms, the PWAS generate in-plane, shear stress interactions with the substrate as depicted in Figure 1b. The resulting Lamb wave motions generate in-plane and out-of-plane motion fields that propagate efficiently away from the sensor location. As depicted in Figure 1b, the inplane, shear stress motions in the PWAS disks actually create out-of-plane motions due to the boundary conditions imposed by the PWAS disk geometry. The circular symmetry of the PWAS disk creates an out-of-plane displacement field that follows a Bessel or Gaussian distribution across the surface of the disk. This is not unlike the displacement field distribution that occurs in traditional NDE contact transducers.

\section{DISPLACEMENT FIELD IMAGING}

A novel displacement-field imaging approach was utilized to understand the vibration characteristics of PWAS transducers. The displacement-field imaging approach is based on a near-field scanning interferometry (NFSI) system that was originally developed for imaging surface-breaking cracks on aerospace material substrates ${ }^{15,16}$. The system uses a scanning laser beam to probe the motions on a material surface using heterodyne interferometry principles. By raster-scanning the probe laser beam relative to the material surface position, a time-resolved motion field can be generated that permits vibrations and elastic wave propagation features to be studied in a non-contact and microscopic fashion. Additional details of the displacement field imaging methodology can be found in reference [16].

An image of the displacement field imaging system is provided in Figure 2a. It utilizes a Polytec OFD heterodyne interferometry system, with positioning, synchronization, and data collection provided by a personal computer running Labview control software. The system has a scan precision of $\sim 1$ micron, a spatial resolution of $\sim 6 \mathrm{~mm}$, a out-of-plane dynamic range of $+/-75 \mathrm{~nm}$, a frequency bandwidth of $20 \mathrm{kHz}-20 \mathrm{MHz}$, and a sensitivity better than 1 nanometer displacement levels.

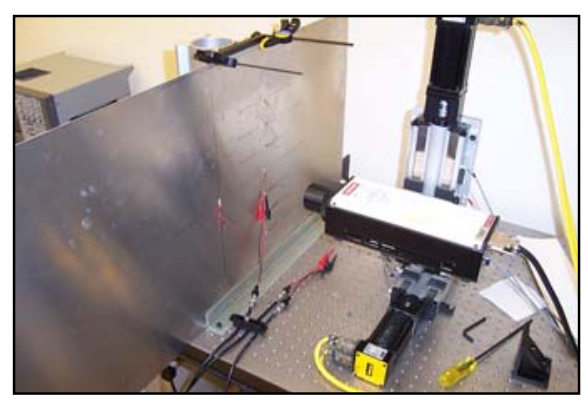

a)

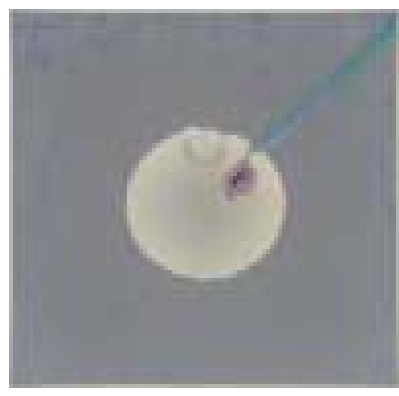

b)
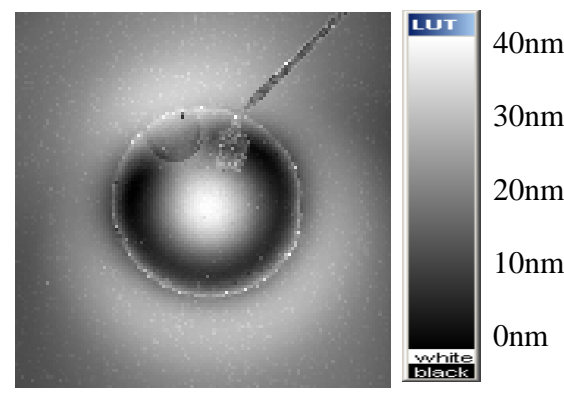

c)

Fig. 2. a) Displacement field imaging system, b) digital image of PWAS, and c) displacement-field image of bonded PWAS.

Figure $2 \mathrm{~b}$ and 2c show a digital and displacement-field image of a PWAS bonded to a thin aluminum plate, respectively. The PWAS was $1 \mathrm{~cm}$ in diameter, and was excited using a $10 \mathrm{Vpp}, 100 \mathrm{kHz}, 1$-cycle impulse from a function generator. The connection wire can be seen in both images extending from the center of the PWAS to the upper right. The displacement field image depicts the time-averaged, peak displacement field for the out-of-plane motions for one vibration cycle. As shown in Figure 2c, the displacement pattern is circularly symmetric and has a peak level of $\sim 40 \mathrm{~nm}$. 


\section{SURVIVABILITY/DURABILITY TESTING OF PWAS}

The main objective of this effort was to study the performance and potential degradation of piezo wafer active sensor (PWAS) systems under adverse mechanical, temperature, and moisture conditions. The displacement-field imaging approach was used to monitor the PWAS motions before and after different environmental exposure events. An important consideration, and point to be stressed, is that the displacement field imaging system uses a laser beam to probe the vibrations of the PWAS and the surrounding material substrate. This means that the measurements are made in a non-contact, and more importantly, non-perturbing manner. This provides a unique opportunity for studying PWAS performance and damage without influencing the integrated system in any way. In addition, the measurements are made with a high spatial resolution (1-10 microns), with an absolute and calibrated sensitivity (1 nanometer), and with a flat frequency response from $20 \mathrm{kHz}$ to $20 \mathrm{MHz}$.

The basic measurement process involved taking a baseline displacement field image scan before environmental exposure, then exposing the bonded PWAS panel to some environmental condition, removing the sample from the exposure conditions, followed by another displacement field image scan. The process was repeated for many environmental exposures in many instances. In all cases, a calibrated and reproducible input voltage signal of 10Vpp, $100 \mathrm{kHz}, 1$-cycle impulse was used for PWAS excitation purposes. This provided a constant input relationship to be compared to the displacement levels measured before and after exposure conditions.

Of particular interest was the detection and imaging of crack events in the PWAS, and disbonding of the PWAS from the aluminum panel substrate. The ability of the displacement field imaging technique to detect and image these types of damage events is depicted in Figure 3a and 3b, where two surface bonded PWAS have disbond and crack indications, respectively. The disbonded PWAS shows lobe intensified regions to the right of the image field, where the disbond event (approximately the entire right half of the PWAS is disbonded) is permitting modal vibrations to occur in the PWAS structure. The vibrations are similar to vibrations that would be present on a vibrating 'drumhead', where the $100 \mathrm{kHz}$ drive frequency determines the spacing, size, and level of vibration energies in the PWAS. The plot depicted in Figure 3c shows the time history of displacement that is occurring at the approximate center of the PWAS, and near the disbonded edge. As shown in the plot, the $100 \mathrm{kHz}$ impulse signal has been replaced by a multi-cycle modal vibration response function.

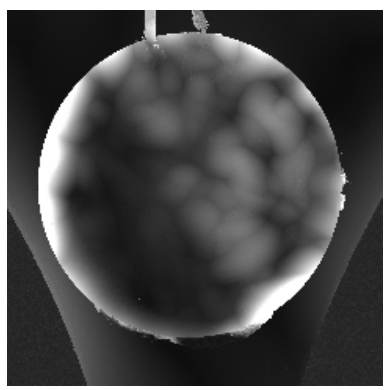

a)

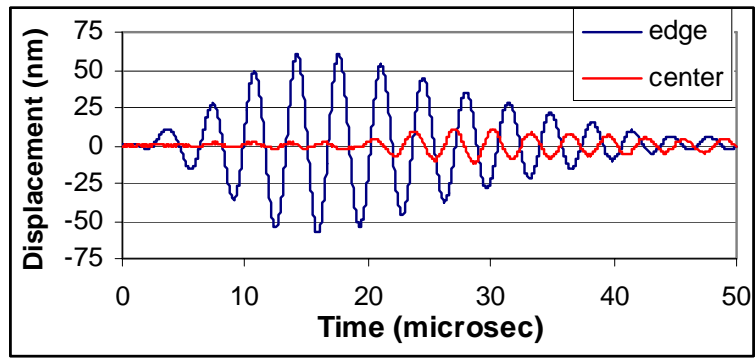

C)

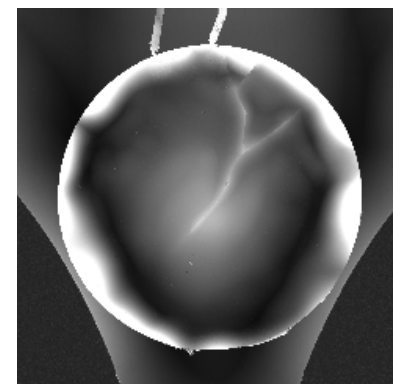

b)

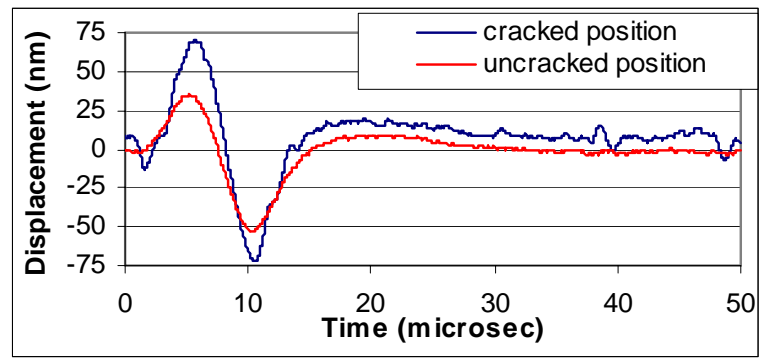

d)

Fig. 3. Displacement field images for a) disbonded PWAS, b) cracked PWAS, and plots for c) disbonded and d) cracked PWAS. 
Figures $3 \mathrm{~b}$ and $3 \mathrm{~d}$ show the displacement field image (time-averaged, peak-to-peak displacement levels) and the time history plot for a PWAS that has been cracked. The PWAS was still bonded to the substrate material, and in fact, the general PWAS displacement pattern and Lamb waves generated by the PWAS were similar to the pre-cracked conditions. The branching crack is easily distinguished in the displacement-field image and is made visible by a nearfield intensification and free-boundary effect described in references [15] and [16]. The displacement levels of the PWAS away from the crack are at the nominal level of 35-40 nm, while the motion levels at and very near the crack are intensified to levels of $\sim 70 \mathrm{~nm}$. This intensification permits cracks and other sharp, free-boundary anomalies to be imaged with the displacement-field imaging technique with relative ease and with a high degree of precision and resolution.

A systematic evaluation of PWAS motion fields was conducted using the displacement field imaging technique to better understand the impact of different environments on the motion field generated by the PWAS. In particular, temperature cycling was investigated, outdoor exposure, electrochemical exposure, and dynamic bending vibrations. In all cases, the PWAS were bonded to the aluminum panels using standard strain gauge bond materials (M-Bond 200 cyanacrylate and catalyst) and preparation procedures specified by the manufacture (Vishay Micro-Measurements).

The temperature cycling experiments were conducted in two separate exposure conditions representative of typical aircraft environments. The first exposure condition involved 12-hour freeze-thaw temperature cycling. For this study, a 12"x12" aluminum panel with a single PWAS bonded in its center was exposed to repeated freeze-thaw conditions in 12- hour cycles. The freeze conditions were applied in a typical commercial freezer environment set to 5F (-15C). After a baseline displacement field image scan was accomplished, the sample was placed in the sub-freezing conditions for 12 hours. After removal, the sample was permitted to thaw to room temperature (75F, 24C), and an additional displacement field image scan was made. Figure 4a shows a representative 12"x12” sample used in the experiment, while Figure 4b depicts four displacement field images taken at 0 cycles, 4 cycles, 8 cycles, and 12 cycles, respectively. Figure 4c provides cross-sectional cut data for all 12 freeze-thaw images, and Figure 4d provides a plot of the peak displacement levels for all 12 freeze-thaw displacement field images.

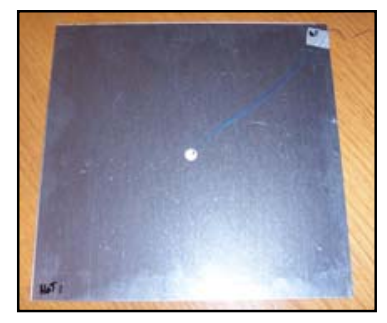

a)

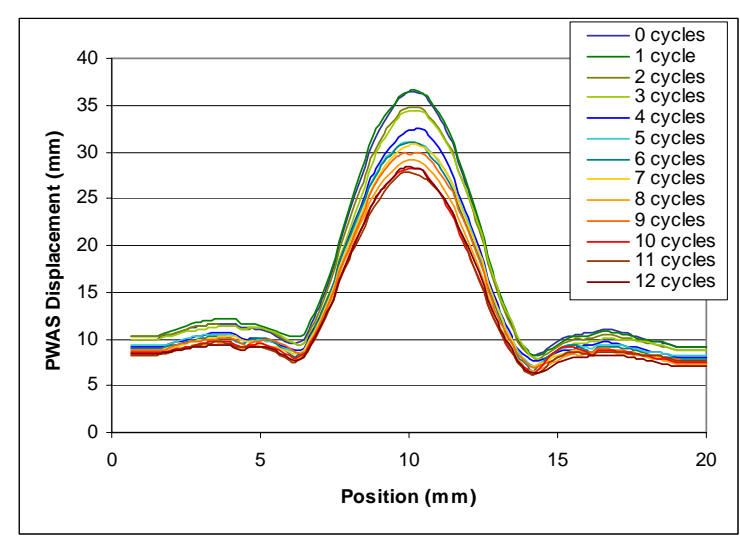

C)

o

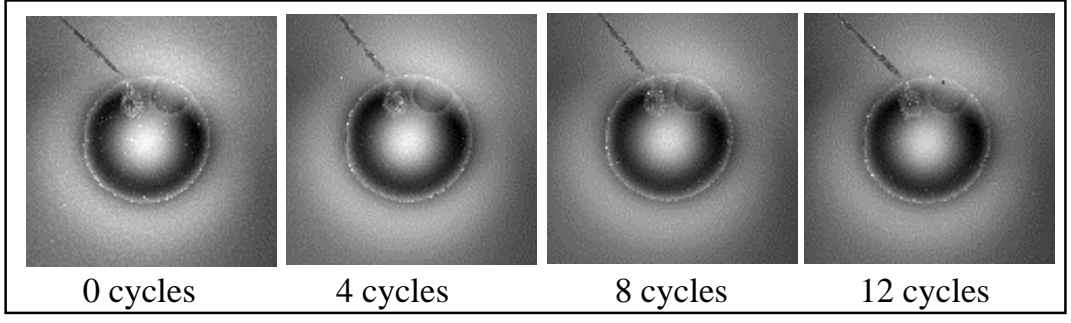

b)

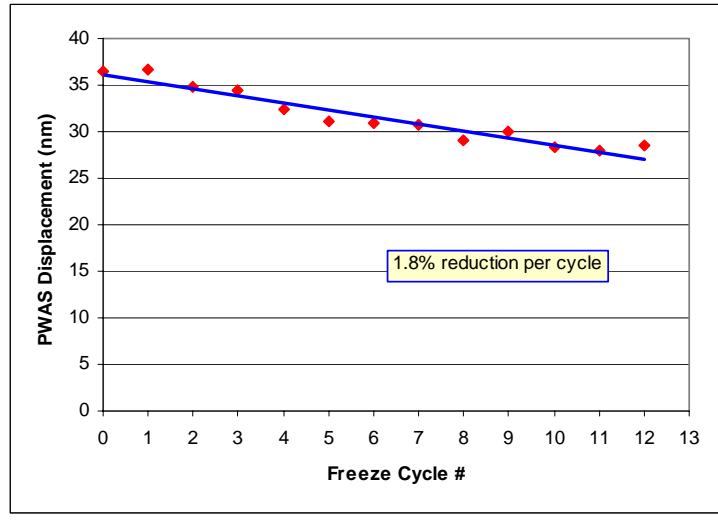

d)

Fig. 4. a) PWAS sample, b) freeze-thaw displacement field images, c) radial displacement cuts, d) peak displacements levels. 
The displacement field images depicted in Figure 4b show no real impact on the basic displacement pattern and structure of the PWAS that would indicate cracking problems of disbond of the PWAS from the substrate material. The plots depicted in Figure 4c and 4d, however, show a general trend in out-of-plane displacement energy levels from $~ 36 \mathrm{~nm}$ peak displacement for 0 cycles of freeze-thaw exposure, to $\sim 28 \mathrm{~nm}$ of displacement after 12 repeated exposures. In fact, the general degradation and loss of vibrational energy resulted in a more-or-less linear trend of $1.8 \%$ reduction in vibration level for each consecutive exposure.

An elevated temperature exposure cycling experiment was also conducted on samples similar to that depicted in Figure 4a. The elevated exposures were for 1-hour at 175F (80C) followed by gradual cooling to room temperature conditions (75F, 24C). Displacement field image scans were taken under room temperature conditions similar to the procedures followed for the freeze-thaw exposures. The displacement field image results are depicted in Figure $5 \mathrm{a}$ for the first four heat exposure cycles. A general darkening trend in the images after each exposure can be seen, which is an indication of a loss in vibration levels for each subsequent heat exposure. This is further shown in the plots depicted in Figure $5 \mathrm{~b}$ and 5c, where the cross-sectional cuts through the center of each image field have been taken and the peak displacement measurements were made, respectively. No indication of disbonding is evident, but a small $1 \mathrm{~mm}$ long crack was noticed after the first heat exposure cycle (see $2^{\text {nd }}$ image in Figure $5 a$ ). The crack appeared to be stable after its initiation. Similar to the freeze-thaw exposure results, the elevated temperature results showed a linear trend in performance reduction with additional heat exposures. The degree of vibration loss was higher, however, and was $\sim 4.7 \%$ per exposure cycle. In addition a more substantial drop was noticed after the first exposure ( $\sim 17 \%$ reduction).

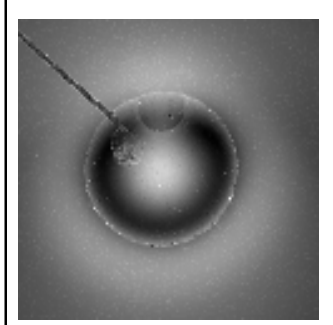

0 cycles

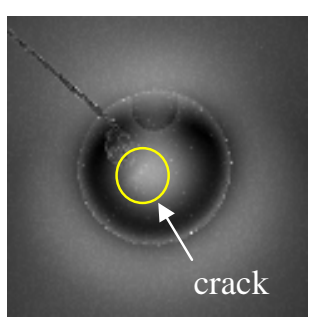

1 cycle

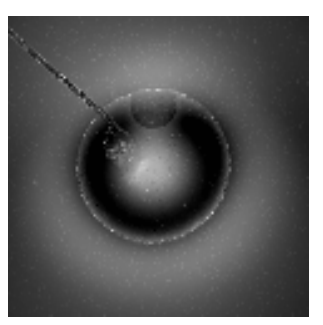

2 cycles

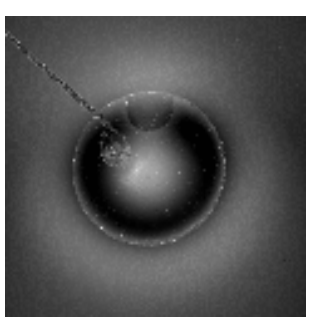

3 cycles

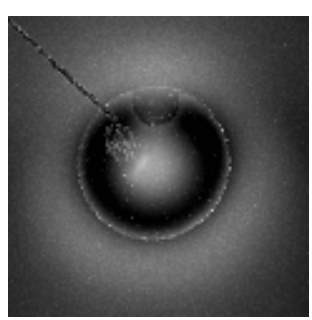

4 cycles

a)

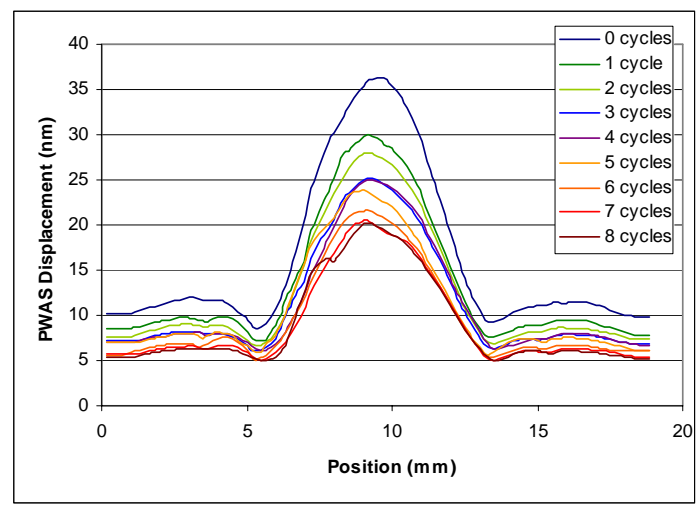

b)

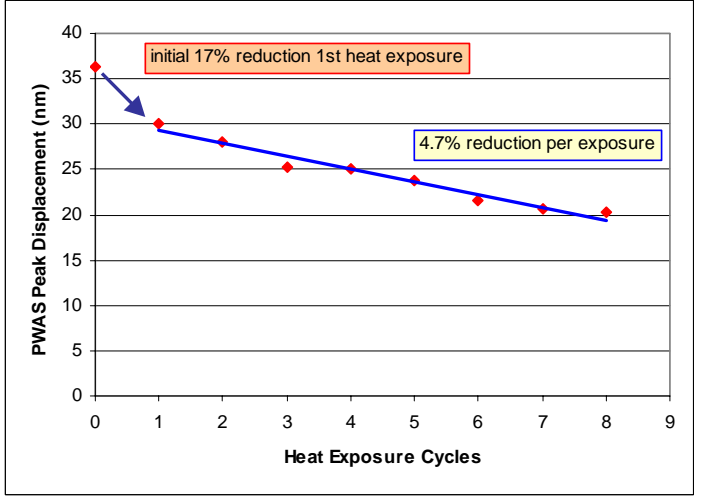

c)

Fig. 5. a) Elevated temperature displacement field images, b) radial displacement cuts, c) peak displacements levels.

A long duration outdoor exposure PWAS sample was also evaluated using the displacement field imaging technique. In this case, the PWAS was mounted in the center of a small, 4" diameter aluminum panel, which was subsequently exposed in an outdoor environment at the University of South Carolina for 63 weeks. The mounted PWAS sample and outdoor test conditions are depicted in Figures 6a and 6b, respectively. 


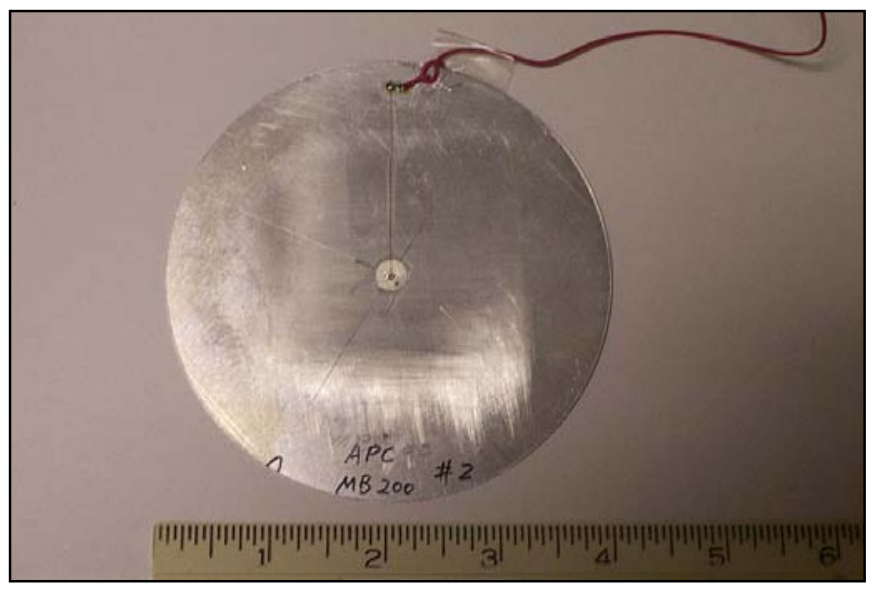

a)

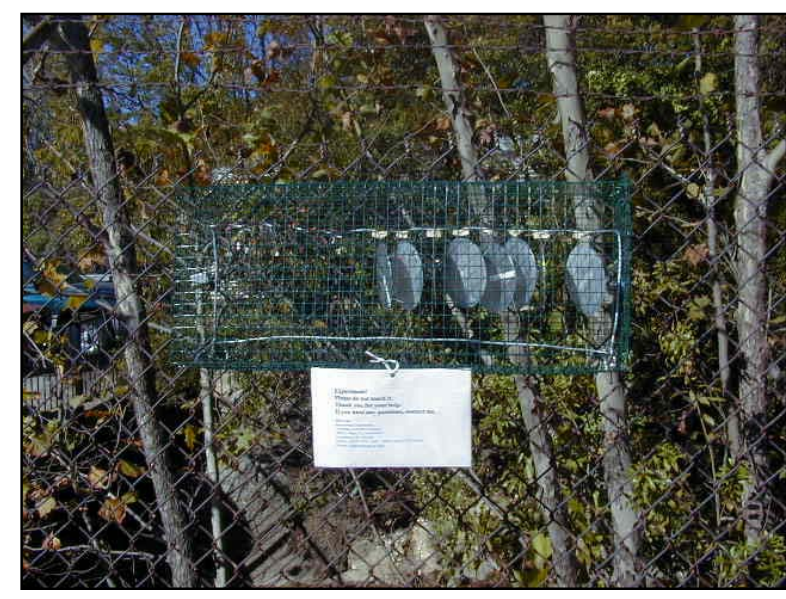

b)

Fig. 6. a) Long-term, outdoor exposure sample, and b) exposure conditions at the University of South Carolina.

The long-term, outdoor exposure sample was compared to a newly-bonded PWAS. Both samples were provided by Dr. Victor Giurgiutiu and James Doane at the University of South Carolina. The displacement field image results for each sample are provided in Figure 7. The unexposed, newly bonded sample image results are provided in Figure 7a, while the 63-week, outdoor exposure sample results are provided in Figure 7b. Two different scan resolutions were taken for each sample including a $7.5 \mathrm{~mm}$ x 7.5mm scan highlighting the PWAS displacements, and a 20mm x 20mm scan highlighting the displacement field propagating away from the PWAS. The 63-week exposed PWAS showed an indication of cracking, edge disbond increases, and a distorted/asymmetric displacement field relative to the newly bonded PWAS sample.

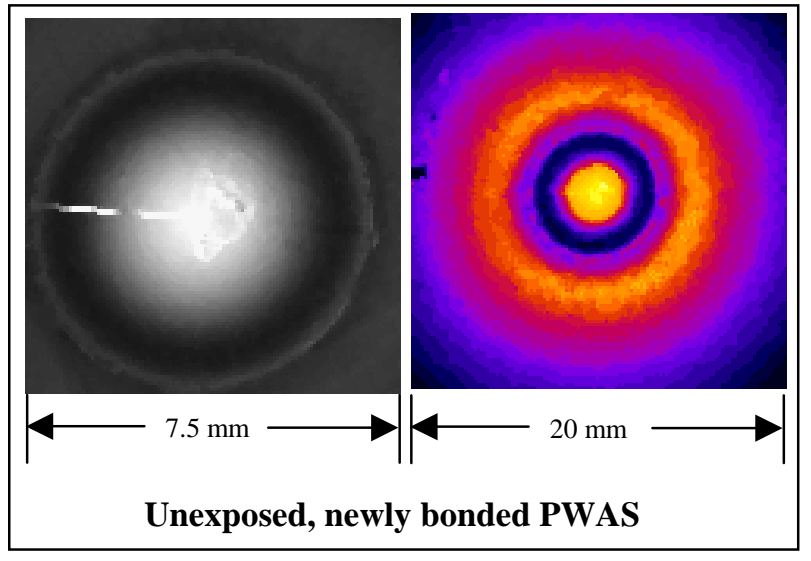

a)

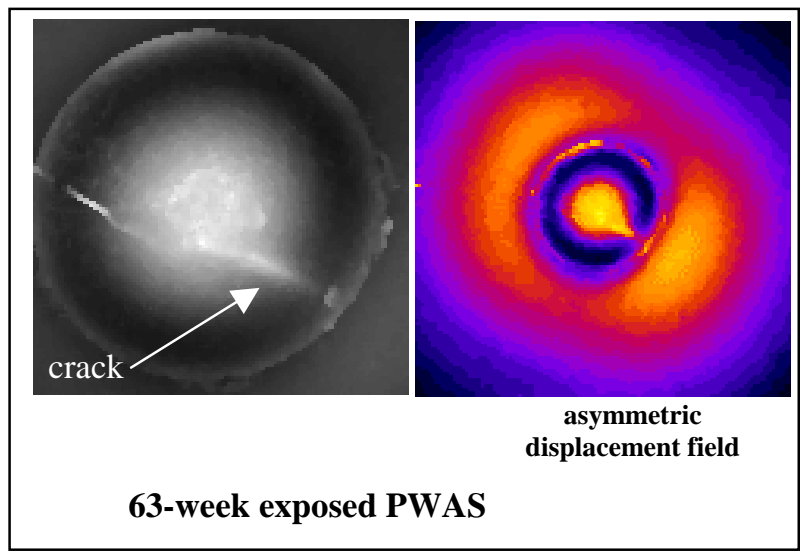

b)

Fig. 7. Displacement field images for a) unexposed, newly bonded PWAS, and b) 63-week outdoor exposed PWAS.

An electrochemically exposed experiment was also conducted. In this experiment, two PWAS sensors were bonded to an aluminum substrate material separated by $\sim 1$ inch distance. One of the PWAS was coated with a water-proof protective enamel coating, while the other PWAS was left unprotected (the wire leads to both PWAS samples were protected. A basic electrochemical immersion cell was then constructed around the perimeter of both sensors. The immersion cell consisted of an electrolyte solution of 3.5\% $\mathrm{NaCl}$ to a depth of approximately $1 \mathrm{~cm}$, and a platinum electrode as shown in Figure 8a. A magnified image of the corrosion cell taken during an electrochemical exposure is shown in Figure 8b. 


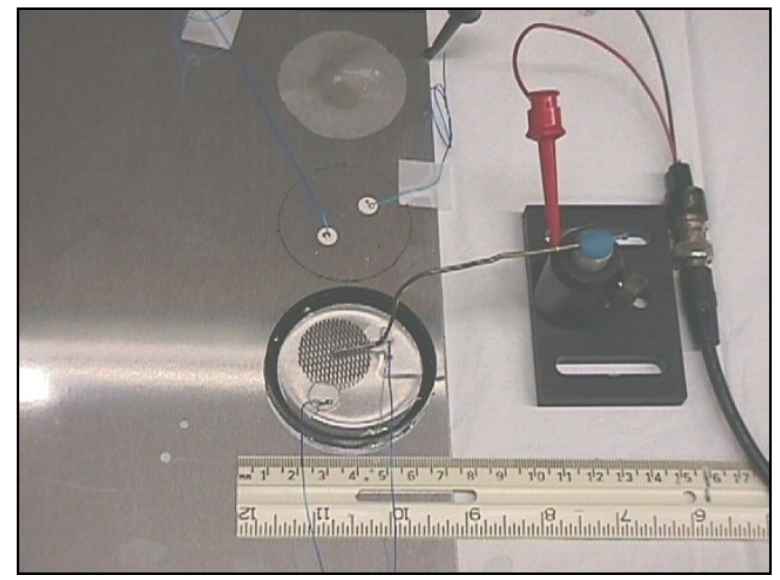

a)

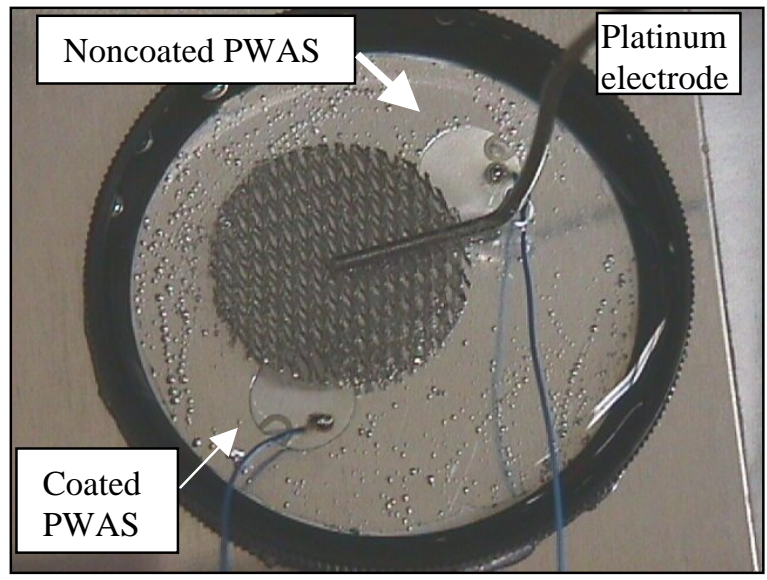

b)

Fig. 8. a) Electrochemical exposure setup, and b) magnified view of electrochemical setup during an exposure cycle.

Figure 9 provides displacement field imaging results for the coated PWAS sensor, while Figure 10 provides results for the noncoated PWAS sensor. Figures 9a and 10a provide displacement images of the unexposed PWAS and the PWAS after four corrosion cell exposures, respectively. As shown in Figures 9b, 9c, 10b, and 10c, there was minimal impact on the PWAS sensor performance, and no observations of cracking or disbond events for seven exposure cycles.

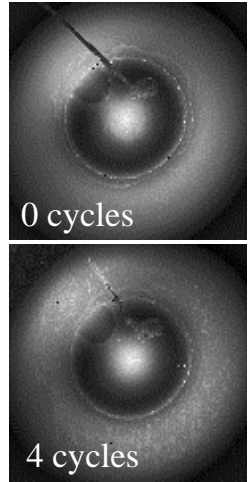

a)

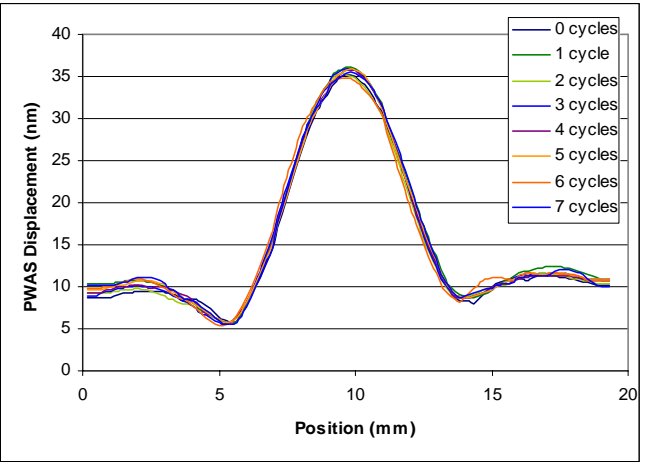

b)

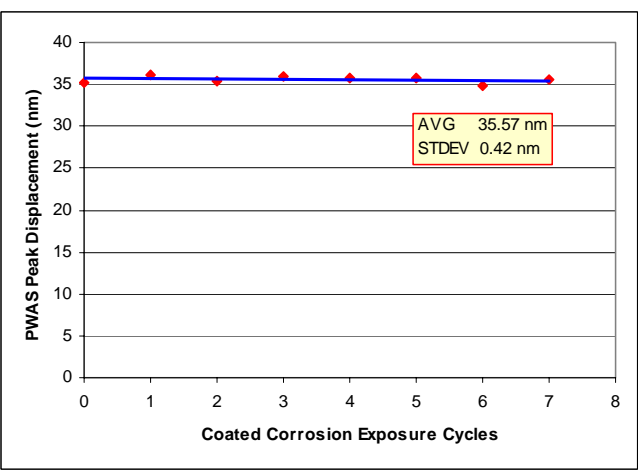

c)

Fig. 9. a) Coated PWAS corrosion cell displacement field images, b) radial displacement cuts, c) peak displacements levels.

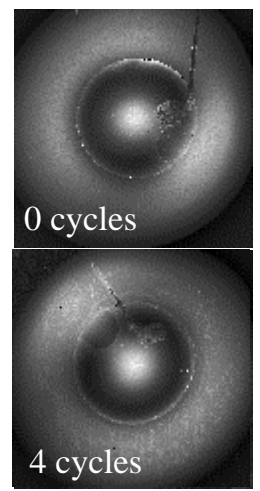

a)

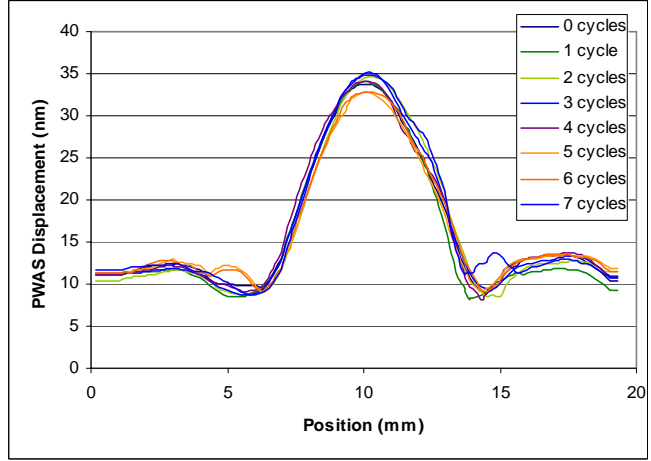

b)

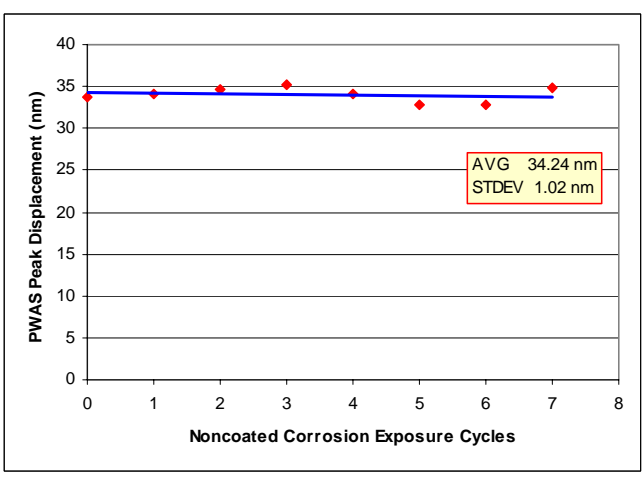

c)

Fig. 10. a) Noncoated PWAS corrosion cell displacement field images, b) radial displacement cuts, c) peak displacements levels. 
On additional measurement was conducted involving the use of a dynamic shaker system with a cantilever bending motion. Five PWAS sensors were bonded to the center region of a thin aluminum plate in a cantilever setup as depicted in Figure 11a. The dynamic shaker system was then used to produce an aggressive and continuous motion field with a nominal frequency of $70 \mathrm{~Hz}$, and a maximum strain level of $2093 \mathrm{uin} / \mathrm{in}$. The displacement field image results for three of the PWAS sensors are provided in Figure 11b and 11c. In Figure 11b, disbond effects can be noticed along with a crack in the lower image. In Figure 11c, an actual fracture of one of the PWAS had occurred with a digital image shown in the top image and the corresponding displacement field image shown in the lower image. It is interesting to note that although all of the PWAS in this test were damaged (disbond, cracking, fracture), in all cases they continued to perform to a certain degree.

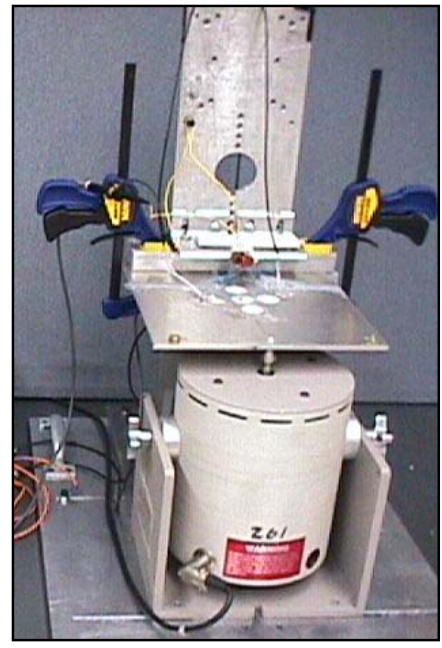

a)
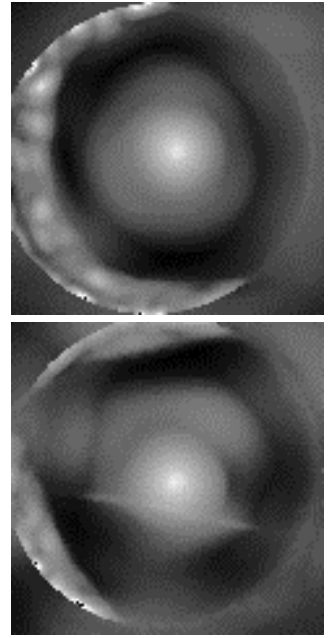

b)

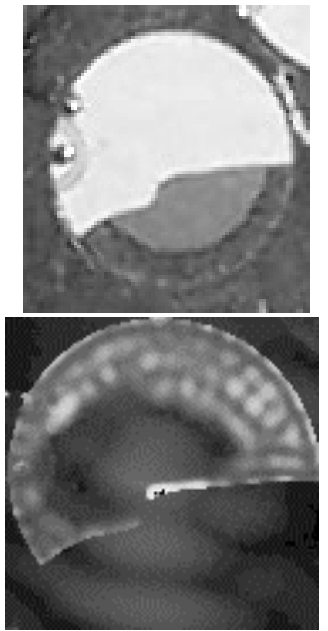

c)

Fig. 11. a) Schematic diagram of fatigue dogbone dimensions, and b) digital image of laser machined notch in gauge region.

\section{CONCLUSIONS}

In this research effort, the long-term operability, durability, and survivability of integrated sensor systems and their associated hardware were evaluated. In particular, the environmental effects of temperature cycling, outdoor exposure, electrochemical exposure, and dynamic vibrations were studied for their impact on integrated piezo wafer active sensors (PWAS). A novel displacement-field imaging approach was utilized to understand the vibration characteristics of PWAS transducers placed in accelerated vibration, temperature-cycling, and moisture-cycling conditions. The results showed damage in the form of PWAS sensor cracking events, bond degradation and failure, as well as indications of performance variation and reduction due to the accelerated exposure levels. Future activities will focus on identifying critical durability and survivability issues through advanced sensor modeling and additional accelerated testing efforts, with the ultimate goal of improving the robustness of health monitoring systems through improved sensor system design and packaging.

\section{REFERENCES}

1. Elgamal, A., Trivedid, M., El Zarkie, M., and Frasera, M., "Health Monitoring Framework for Bridges \& Civil Infrastructure," in Structural Health Monitoring 2003, Fu-Kuo Chang (Ed), Technomonic, 2003, pp. 123-130.

2. Dalton, R., Cawley, P., and Lowe, M., "The Potential of Guided Waves for Monitoring Large Areas of Metallic Aircraft Fuselage Structures,” Journal of Nondestructive Evaluation, Vol. 20, 2001, pp. 29-45.

3. Ihn, J., and Chang, F.-K., "Build-in Diagnostics for Monitoring Crack Growth in Aircraft Structures," Proceedings of SPIE's $9^{\text {th }}$ International Symposium on Smart Structures and Materials, March 2002, San Diego, CA. 
4. Trego, A., "Installation of the Autonomous Structural Integrity Monitoring System," in Structural Health Monitoring 2003, Fu-Kuo Chang (Ed), Technomonic, 2003, pp. 863-870.

5. Raich, A., and Liszkai, T., "Multi-Objective Genetic Algorithms for Optimizing Sensor Layouts to Enhance Structural Damage Identification,” in Structural Health Monitoring 2003, Fu-Kuo Chang (Ed), Technomonic, 2003, pp. 650-657.

6. Lee, B., and Staszewski, E., "Modeling of Lamb Waves for Damage Detection in Metallic Structures: Part I Wave Propagation,” Smart Materials and Structures, Vol. 12, 2003., pp. 804-814.

7. Lee, B., and Staszewski, E., "Modeling of Lamb Waves for Damage Detection in Metallic Structures: Part II Wave Interactions with Damage,” Smart Materials and Structures, Vol. 12, 2003., pp. 815-824.

8. Basheer, M., Derriso, M., and Rao, V., "Self Organizing Wireless Sensor Networks for Structural Health Monitoring,” in Structural Health Monitoring 2003, Fu-Kuo Chang (Ed), Technomonic, 2003, pp. 1193-1207.

9. Giurgiutiu, V., Zagrai, A., Bao, J., Redmond, J., Roach, D., and Rackow, K., "Active Sensors for Health Monitoring of Aging Aerospace Structures, International Journal of the Condition Monitoring and Diagnostic Engineering Management, Vol. 6, No. 1, 2003, pp. 3-21.

10. Wang, C., and Chang, F.-K., "Built-In Diagnostics for Impact Damage Identification of Composite Structures," in Structural Health Monitoring 2000, Fu-Kuo Chang (Ed), Technomonic, 2000, pp. 612-621.

11. Koh, Y., and Chiu, W., "Numerical Study of Detection of Disbond Growth under a Composite Repair Patch”, Smart Materials and Structures, Vol. 12, 2003, pp. 633-641.

12. Giurgiutiu, V., Zagrai, A., and Bao, J., "Piezoelectric Wafer Embedded Active Sensors for Aging Aircraft Structural Health Monitoring, International Journal of Structural Health Monitoring, Vol. 1, 2002, pp. 41-61.

13. Sohn, H., and Farrar, C., "Damage Diagnosis Using Time Series Analysis of Vibration Signals," Smart Materials and Structures, Vol. 10, 2001, pp. 1-6.

14. Park, G., Sohn, H., Farrar, C., and Inman, D., "Overview of Piezoelectric Impedance-Based Health Monitoring and Path Forward,” The Shock and Vibration Digest, Vol. 35, No. 6, 2003, pp. 451-463.

15. Blackshire, J., and Sathish, S., "Near-field ultrasonic scattering from surface-breaking cracks," Applied Physics Letters, Vol. 80, 2002, pp. 3442-3444.

16. Blackshire, J., "Interferometric and Holographic Imaging of Surface Wave Patterns for Characterization of Material Degradation," Nondestructive Materials Characterization with Applications to Aircraft Materials, N. Meyendorf, P. Nagy, and S. Rokhlin editors, Springer-Verlag, New York, 2003, pp. 113-140. 